Series A

I. MATHEMATICA

$336 / 10$

\title{
NON_NEGATIVITY PROPERTIES OF OPERATORS IN SPACES WITH INDEFINITE METRIC
}

BY

JÁNOS BOGNÁR 
Communicated 8 March 1963 by P. J. Myrberg and Olli Leh'o 


\section{Non-negativity properties of operators in spaces with indefinite metric}

1. The following statements concerning a bounded self-adjoint operator $A$ on a Hilbert space $H$ are known to be equivalent:

a. $(A x, x) \geqq 0$ for every $x \in H$.

b. $A=C^{*} C$ with some bounded linear operator $C$.

c. $A=B^{2}$ where $B$ is a bounded self-adjoint operator.

d. The spectrum of $A$ contains non-negative values only.

e. For sufficiently small positive numbers $\alpha$ there exists a projector $\boldsymbol{P}(\alpha)$ in a wider Hilbert space $\boldsymbol{H}(\alpha)$ such that $\alpha A$ is the projection of the operator $\boldsymbol{P}(\alpha)$ onto the original space $H$ (cf. [6], p. 443).

Operators $A$ fulfilling the conditions a-e are said to be non-negative.

Now let $H$ be a linear space with a hermitian bilinear sinner product» $(x, y)$, the form $(x, x)$ being not necessarily definite. Let $A$ denote a linear operator, defined everywhere in this space and satisfying $(A x, y)=(x, A y)$ for every pair of elements $x, y \in H$. For such an operator one may try to introduce the notion of mnon-negativeness with respect to the space $H$ " by generalizing the conditions a-e. But the conditions a - e are five in number, and some of them have several "natural» generalizations. Which one should we choose? If we could find a property, generalizing at the same time all the properties a-e (or so many of them as possible), we might expect that this property would provide us a useful notion of non-negativeness.

Thus we are led to the problem of finding generalizations of the conditions $\mathrm{a}-\mathrm{e}$ to the case of more general inner product space: than Hilbert space, and of studying their logical connections.

In the present paper we make a step in this direction. We restrict our attention to the class of $H_{k}$ spaces $(k=0,1,2, \ldots)$, i. e. direct products of a Hilbert space and a $k$-dimensional negative unitary space. It is evident that $H_{0}$ space and Hilbert space are the same thing, so that our class contains all Hilbert spaces and we may speak about generalizations of the conditions a-e to this class of spaces. On the other hand, in the case of $H_{k}$ spaces the necessary definitions and lemmas are at our disposal in works of L. S. Pontrjagin [5] and I. S. Iohvidov-M. G. Kreinn [4].

So we are going to examine non-negativity properties of operators in $H_{k}$. We list the proposed generalizations of $\mathrm{a}-\mathrm{e}$, announce some impli- 
cations between them, and give examples which show the failure of some other implications. For several pairs of properties the question whether one implies the other remains open.

The proofs of the stated implications will appear in [2].

2. In this section we recall some notions and facts concerning $H_{k}$ spaces and their operators (cf. [5], [4]).

We consider a complex linear space $H$ together with a hermitian bilinear functional $(x, y)$ defined for every pair of elements $x, y \in H$.

An element $x$ is called positive (resp. negative, non-negative, non-positive, neutral) if $(x, x)>0 \quad$ (resp. $\quad(x, x)<0, \quad(x, x) \geqq 0, \quad(x, x) \leqq 0$, $(x, x)=0)$. A linear subspace is said to be positive (resp. negative, nonnegative, non-positive, neutral) if all its elements, with the possible exception of the vector 0 , have the respective property.

Two elements $x, y$ are said to be orthogonal if $(x, y)=0$. The de. finition of the orthogonality of a vector to a subspace or of two subspaces to each other is evident. The orthogonal complement of a subspace $M$ consists of all elements which are orthogonal to $M$.

$H$ is a space of type $H_{k}$ or an $H_{k}$ space if it has the following three properties (cf. [4], p. 371):

I. $H$ contains a negative subspace of dimension $k$, but $H$ contains no negative subspace of dimension $k+1$.

II. 0 is the only vector orthogonal to the whole space $H$.

III. The $k$-dimensional negative subspace $H^{-} \subset H$ can be chosen so that its orthogonal complement $H^{+}$(which is a positive linear subspace by $\mathrm{I}$ and $\mathrm{II})$ is complete with respect to the norm $\left\|x^{+}\right\|=\left(x^{+}, x^{+}\right)^{1 / 2}$ $\left(x^{+} \in H^{+}\right)$.

Accordingly, an $H_{k}$ space is the orthogonal direct sum of a megative unitary space» of dimension $k$ and a Hilbert space of arbitrary dimension ${ }^{1}$ ).

It can be shown ([4], pp. $375-377)$ that in an $H_{k}$ space III is satisfied for every $k$-dimensional negative subspace $H^{-}$, and the Hilbert-norms

$$
\|x\|=\sqrt{-\left(x^{-}, x^{-}\right)+\left(x^{+}, x^{+}\right)} \quad\left(x=x^{-}+x^{+} ; x^{-} \in H^{-}, x^{+} \in H^{+}\right) .
$$

corresponding to different direct decompositions $H_{k}=H^{-} \oplus H^{+}$, induce the same notion of strong convergence. Consequently, the notions of closed subspace, bounded operator, spectrum of an operator etc., introduced with the aid of one of these norms, are independent of the choice of the subspace $H^{-}$.

1) In [4] the symbol $\Pi_{\%}$ is used instead of $H_{k}$, and the roles of positive and negative subspaces are interchanged in the definition. 
The adjoint $T^{*}$ of a bounded linear operator $T$ in $H_{k}$ is defined by $(T x, y)=\left(x, T^{*} y\right) \quad\left(x, y \in H_{k}\right)$. The operator $T$ is self-adjoint if $T^{*}=T$. The self-adjoint operator $P$ is a projector if $P^{2}=P$. Projectors have a geometrical meaning analogous to that in Hilbert space, apart from the fact that in $H_{k}$ projectors can be associated only with those closed subspaces which contain no vector $x_{0} \neq 0$ orthogonal to the whole subspace ([5], p. 255). This is in connection with the fact that a closed subspace and its orthogonal complement in $H_{k}$ do not span, as a rule, the space, and they can have common elements other than 0 .

We mention two important theorems concerning self-adjoint operators in $H_{k}$. The first asserts that any of these operators has a $k$-dimensional, non-positive, invariant subspace ([5], p. 257). According to the second, the spectrum of a self-adjoint operator in $H_{k}$ lies on the real axis, excepting at most $k$ conjugate complex pairs of proper values ([4], p. 424).

3. In the following $A$ denotes a bounded, self-adjoint operator in a space $H_{k}$.

For the sake of brevity we shall say that the subspace $M \subset H_{k}$ is $A$-positive (resp. $A$-negative, $A$-non-negative, $A$-non-positive, $A$-neutral), if for every element $x \in M(x \neq 0)$ we have $(A x, x)>0$ (resp. $(A x, x)<0$, $(A x, x) \geqq 0, \quad(A x, x) \leqq 0, \quad(A x, x)=0)$.

Using these terms, the following generalizations of the conditions a-e (cf. Section 1) seem to be natural.

a 1. The whole space is $A$-non-negative.

a 2. No subspace of dimension $k+1$ is $A$-negative.

a 3. There exists a $k$-dimensional $A$-non-positive subspace, but no subspace of dimension $k+1$ is $A$-negative.

a 4. There exists a $k$-dimensional $A$-non-positive subspace with $A$-non-negative orthogonal complement.

a 5. There exists a $k$-dimensional, non-positive, $A$-non-positive subspace, but no subspace of dimension $k+1$ is $A$-negative.

a 6 . There exists a $k$-dimensional, non-positive, $A$-non-positive subspace with $A$-non-negative orthogonal complement.

a 7 . There exists a $k$-dimensional, negative, $A$-non-positive subspace, but no subspace of dimension $k+1$ is $A$-negative.

a 8 . There exists a $k$-dimensional negative, $A$-non-positive subspace with $A$-non-negative orthogonal complement.

a 9 . $A$ has a $k$-dimensional, non-positive, $A$-non-positive, invariant subspace, but no subspace of dimension $k+1$ is $A$-negative.

a 10. $A$ has a $k$-dimensional, non-positive, $A$-non-positive, invariant subspace with $A$-non-negative orthogonal complement. 
a 11. $A$ has a $k$-dimensional, negative, $A$-non-positive, invariant subspace, but no subspace of dimension $k+1$ is $A$-negative.

a 12. $A$ has a $k$-dimensional, negative, $A$-non-positive, invariant subspace with $A$-non-negative orthogonal complement.

a 13. Every non-negative vector is $A$-non-negative; every non-positive vector is $A$-non-positive.

b 1. There exists a bounded operator $C$ such that $A=C^{*} C$.

c 1. There exists a bounded, self-adjoint operator $B$ such that $A=B^{2}$.

d 1. The real values in the spectrum of $A$ are non-negative.

d 2. The spectrum of $A$ contains real, non-negative values only.

e 1. For sufficiently small positive numbers $\alpha$ there exists a projector $\boldsymbol{P}(\alpha)$ in a space $\boldsymbol{H}_{2 k}(\alpha)$, containing the original space $H_{k}$ as a closed subspace, such that $\alpha A$ is the projection of the operator $\boldsymbol{P}(\alpha)$ onto $H_{k}$.

e 2. For sufficiently small positive numbers $\alpha$ there exists a projector $\boldsymbol{P}(\alpha)$ in a space $\boldsymbol{H}_{k}(\alpha)$, containing the original space $H_{k}$ as a closed subspace, such that $\alpha A$ is the projection of the operator $\boldsymbol{P}(\alpha)$ onto $H_{k}$.

4. It is easy to see that in the special case $k=0$ the "non-negativity properties", listed in the previous section, really reduce to the corresponding Hilbert space properties (Section 1). Consequently, in this special case they are all equivalent. Now we shall study their inter-relations in the nontrivial case $k>0$.

It is evident that a 1 implies a 2 ; a 3 implies a 2 ; a 5 implies a 3 ; a 6 implies a 4; a 7 implies a 5; a 8 implies a 6 ; a 9 implies a 5; a 10 implies a 6 ; a 11 implies a 7; a 11 implies a 9; a 12 implies a 8; a 12 implies a 10; c 1 implies b 1 ; d 2 implies d 1 .

It is also not difficult to show that a 4 implies a 3 ; a 6 implies a 5 ; a 8 implies a 7; a 10 implies a 9; a 12 implies a 11 ; a 12 implies $d 2$; b 1 implies a 2; a 12 implies c 1; c 1 implies a 10. (Stronger versions of the last two assertions have been proved in [1].)

Something more interesting are the following relations: a 11 implies a 4 ; a 13 implies a 12 ; e 1 is equivalent to a 2 ; e 2 is equivalent to a 1 .

All these relations are indicated on Fig. 1.

For the special case of $H_{1}$ spaces we are also able to prove that a 3 implies a 4 , and a 6 implies a 10 . For general $k$ we do not know whether these statements are true.

5. We give some examples which restrict the number of possible further implications between the "non-negativity properties", and at the same time show how much more complicated the situation is in $H_{k}$ spaces $(k>0)$ than in Hilbert space. 


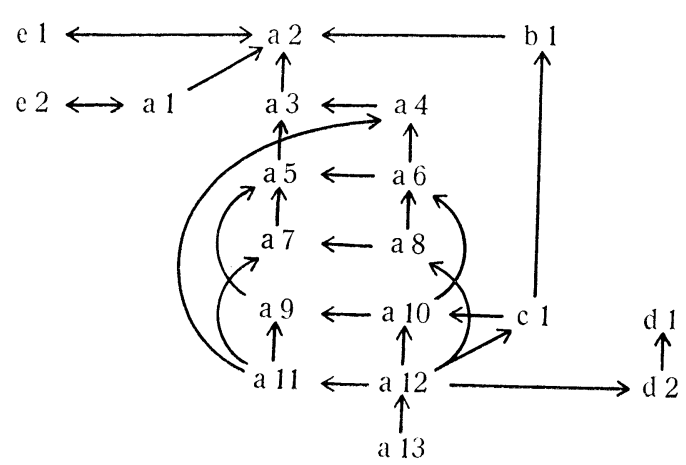

Fig. 1

We consider the $H_{1}$ space spanned by two vectors $e_{1}, e_{2}$ satisfying $\left(e_{1}, e_{1}\right)=-1,\left(e_{2}, e_{2}\right)=1,\left(e_{1}, e_{2}\right)=0$. Keeping in mind that a linear operator in this space is self-adjoint if and only if its matrix $\left(a_{i j}\right)$, belonging to the basis $e_{1}, e_{2}$, fulfils the conditions $a_{11}=\bar{a}_{11}, a_{21}=-\bar{a}_{12}, a_{22}=$ $\bar{a}_{22}$, the following statements are easy to verify.

$\left(\begin{array}{ll}1 & 0 \\ 0 & 1\end{array}\right) \quad$ satisfies a 13,

$\left(\begin{array}{ll}1 & 0 \\ 0 & 2\end{array}\right) \quad$ satisfies a 12 ,

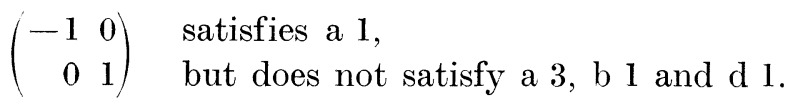

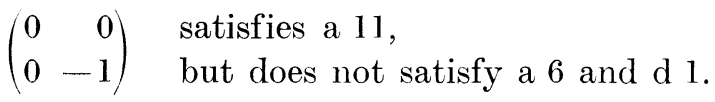

$\left(\begin{array}{rr}-1 & 0 \\ 0 & -1\end{array}\right)$ satisfies c 1 ,

$\left(\begin{array}{rr}-2 & 0 \\ 0 & -1\end{array}\right)$ satisfies a 4 ,

$\left(\begin{array}{rr}-1 & 0 \\ 0 & -2\end{array}\right)$ satisfies a 7 ,

$\left(\begin{array}{ll}1 & -1 \\ 1 & -1\end{array}\right) \quad \begin{aligned} & \text { satisfies a } 10 \text { and } d 2, \\ & \text { but does not satisfy a } 11 \text { and } \mathrm{c} 1 .\end{aligned}$

$\left(\begin{array}{ll}0 & i \\ i & 0\end{array}\right) \quad$ satisfies a 8 and $\mathrm{d} 1$,

Our last example is an operator in the $H_{1}$ space of the square-summable sequences of complex numbers, the inner product of two elements $x=$ $\left\{\xi_{1}, \xi_{2}, \ldots\right\}, y=\left\{\eta_{1}, \eta_{2}, \ldots\right\}$ being defined by 


$$
(x, y)=-\xi_{1} \bar{\eta}_{1}+\sum_{n=2}^{\infty} \xi_{n} \bar{\eta}_{n}
$$

Let $C$ denote the shift operator: $C\left\{\xi_{1}, \xi_{2}, \ldots\right\}=\left\{0, \xi_{1}, \xi_{2}, \ldots\right\}$. Then $C^{*}\left\{\eta_{1}, \eta_{2}, \ldots\right\}=\left\{-\eta_{2}, \eta_{3}, \ldots\right\}$, and $\left(C^{*} C x, x\right)$ is positive definite. Hence b 1 does not imply a 3 .

Other non-implications may be obtained with the aid of the relations in Section 4. For instance, from the last example it follows (cf. Fig. 1) that $\mathrm{b} 1$ does not imply $\mathrm{c} 1$.

The unsolved questions are not many, but interesting. If the propositions "a 3 implies a 4», "a 6 implies a 10», stated here for $k=1$, could be proved for general $k$, all the inter-relations of the fifteen conditions a $1-$ a 13 , e 1 - e 2 would be known. However, in the course of further study of b 1, c $1, \mathrm{~d} 1$ and $\mathrm{d} 2$ the introduction of new "non-negativity properties" may turn out to be reasonable.

Finally we mention two theorems of Ju. P. Ginzburg ([3], pp. 15-16), proved for more general spaces than $H_{k}$, which have something to do with our results: 1. If the whole space is $A$-non-negative, then the spectrum of $A$ is real. 2. If the spectrum of $A$ is positive, then $A$ has a self-adjoint square-root.

Hungarian Academy of Sciences

Budapest, Hungary 


\section{References}

[1] BogNÁR, J.: О существовании квадратного корня из оператора, самосопряженного относительно индефинитной метрики. Abstract: On the existence of square roots of operators, selfadjoint with respect to an indefinite metric. - Magyar Tud. Akad. Mat. Kutató Int. Közl. 6, 1961, pp. 351-363.

[2] -»- О некоторых соотношениях между свойствами неотрицательности операторов в пространствах с индефинитной метрикой. Abstract: Some connections between non-negativity properties of operators in spaces with indefinite metric. - To appear in Magyar Tud. Akad. Mat. Kutató Int. Közl. $8,1963$.

[3] Гинзьург, Ю. П.: О J-нерастягивающих операторах в гильбертовом пространстве. - Научные Записки Физико-Математического Факультета Одесского Педагогического Института 22: 1, 1958, pp. 13-20.

[4] Иохвидов, И. С., and М. Г. Крейн: Спектральная теория операторов в пространствах с индефинитной метрикой. I. - Труды Москов. Мат. Общ. 5, 1956, pp. 367-432.

[5] Понтрягин, Л. С.: Эрмитовы операторы в пространстве с индефинитной метрикой. - Изв. Акад. Наук СССР. Сер. Мат. 8, 1944, pp. 243-280.

[6] Sz.-NAGY, B.: Prolongements des transformations de l'espace de Hilbert qui sortent de cet espace. - Appendix to the 3rd edition of the book "Leçons d'analyse fonctionnelle» by F. RiEsz and B. Sz.-NAGY, Akadémiai Kiadó, Budapest, 1955. 